\title{
三唑类白杨素衍生物的设计、合成及其抗增殖活性研究
}

\author{
栾 天 ${ }^{a, b, c} \quad$ 全哲山 ${ }^{b} \quad$ 方应权 $^{a}$ 杨宏静 $* a$ \\ ( ${ }^{a}$ 重庆三峡医药高等专科学校药学院 重庆 404120) \\ ( ${ }^{b}$ 延边大学药学院 吉林延吉 133002$)$ \\ (c沈阳医学院药学院 沈阳 110034)
}

\begin{abstract}
摘要 合成了 2 个系列的白杨素衍生物, 采用噻唑蓝(MTT)法测试了所有化合物针对六种肿瘤细胞的体外抗增殖活性, 包括 MGC-803, BEL-7402, HepG2, HeLa, A549 以及 SGC-7901 细胞. 实验结果显示, 7-[1-(3-氟苯基)-1H-1,2,3-三唑-4-甲 氧基]-白杨素(1c)与 7-[1-(2-氯苯基)-1H-1,2,3-三唑-4-甲氧基]-白杨素(1g)针对 MGC-803 细胞的活性与先导化合物白杨 素及阳性对照药 5-氟尿嘧啶相比显著提高. 因此, 化合物 $1 \mathrm{c}$ 与 $1 \mathrm{~g}$ 具有深入研究用以开发抗癌药物的潜能.
\end{abstract}

关键词 白杨素; 合成; 三唑; 衍生物; 抗增殖

\section{Design, Synthesis and Antiproliferative Activity of Chrysin Derivatives Bearing Triazole Moieties}

\author{
Luan, Tian ${ }^{a, b, c} \quad$ Quan, Zheshan $^{b} \quad$ Fang, Yingquan $^{a} \quad$ Yang, Hongjing*,a \\ ( ${ }^{a}$ Department of Pharmacy, Chongqing Three Gorges Medical College, Chongqing 404120) \\ ( ${ }^{b}$ College of Pharmacy, Yanbian University, Yanji, Jilin 133002) \\ ( ${ }^{c}$ College of Pharmacy, Shenyang Medical College, Shenyang 110034)
}

\begin{abstract}
Two series of novel chrysin derivatives were synthesized, and their antiproliferative activity was evaluated against six human cancer cell lines (MGC-803, BEL-7402, HepG2, HeLa, A549, and SGC-7901) using methyl thiazolyl tetrazolium (MTT) assay. Preliminary bioassay results indicated that 7-((1-(3-fluorophenyl)-1H-1,2,3-triazol-4-yl)methoxy)-5-hydroxy2-phenyl-4H-chromen-4-one (1c) and 7-((1-(2-chlorophenyl)-1H-1,2,3-triazol-4-yl)methoxy)-5-hydroxy-2-phenyl-4H-chromen-4-one (1g) exhibited significantly improved antiproliferative activities against the MGC-803 cell line when compared with the parent compound chrysin and the positive control drug 5-fluorouracil. It demonstrates that compounds $\mathbf{1 c}$ and $\mathbf{1 g}$ are potential agents for cancer therapy.

Keywords chrysin; synthesis; triazole; derivatives; antiproliferation
\end{abstract}

\section{Introduction}

Chrysin is a flavonoid compound extracted from the Senmen oroxyli of the genus Dictyophora. It exhibits a wide range of biological activities including antioxidant, ${ }^{[1]}$ antiviral, ${ }^{[2]}$ antiallergy, ${ }^{[3]}$ anti-inflammatory, ${ }^{[4]}$ antianxiety $^{[5]}$ and antiproliferative ${ }^{[6-9]}$ effects. The antiproliferative activities of chrysin and its derivatives have attracted considerable attention in the recent years. Other biochemical properties of chrysin, such as low solubility in vitro and low bioavailability in vivo, restrict its clinical application. ${ }^{[10,11]}$ Therefore, the discovery of highly active derivatives via the structural modification of chrysin during the development of antiproliferative agents is promising.

Triazole fragments are important pharmacophores that display diverse biological functions, especially antiproliferative activities. The introduction of triazole fragments can improve the pharmacokinetic property, and biological activities of the parent compounds. ${ }^{[12]}$ Many reports have shown that introducing 1,2,3-triazole or 1,2,4-triazole fragments in different natural products can improve their antiproliferative activities. For example, compound A (Figure 1) exhibited a significantly improved antiproliferative activity when compared with the parent compound. ${ }^{[13]}$ Furthermore, Liu et al. ${ }^{[14]}$ reported a series of compounds linked to 1,2,4-triazole, and as the result, compound $\mathbf{B}$

* Corresponding author. E-mail: 40278667@qq.com

Received July 5, 2019; revised July 23, 2019; published online October 12, 2019.

Project supported by the National Natural Science Foundation of China (No. 21662036).

国家自然科学基金(No. 21662036)资助项目. 


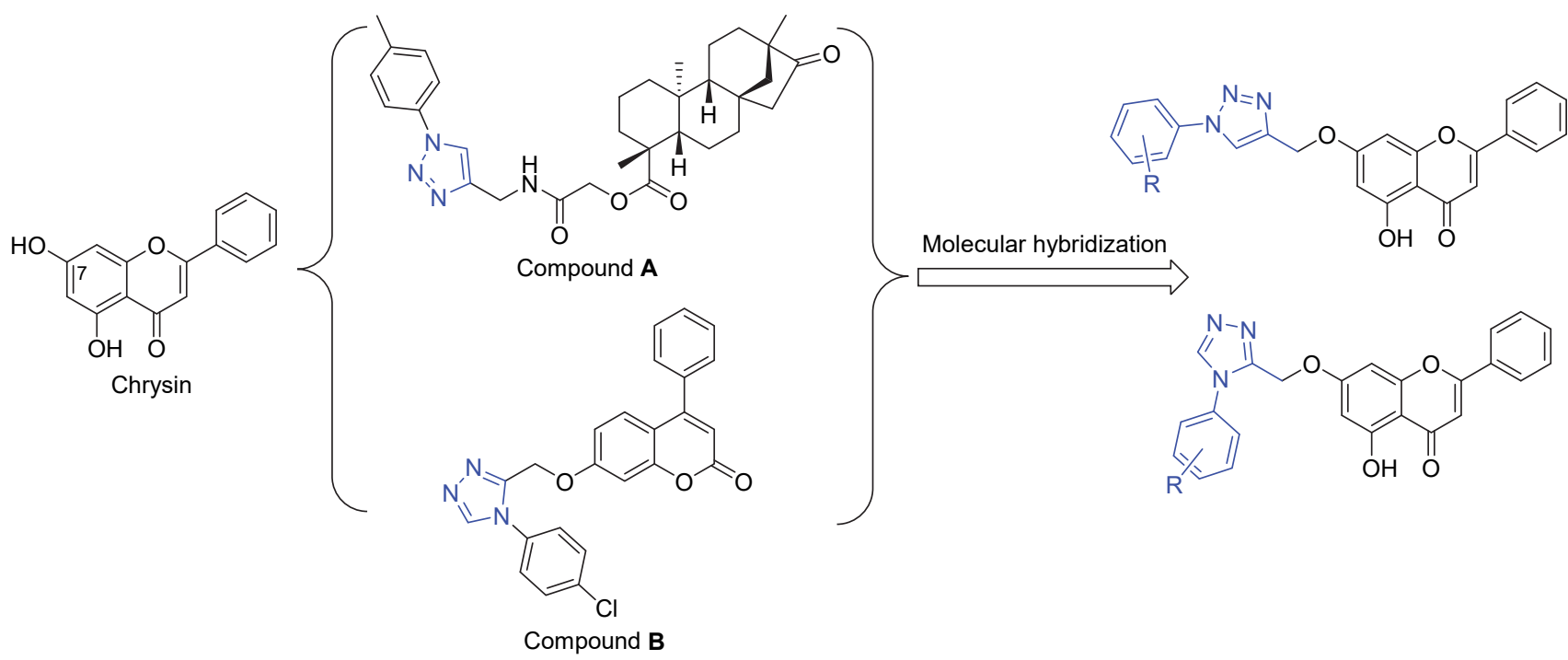

Figure 1 Design of target compounds based on the combination principles

showed promising antiproliferative activities against different types of cancer cell lines.

Previous studies showed that the structural modification in the 7 hydroxyl group can improve the bioactivity of chrysin. ${ }^{[15]}$ In this study, we synthesized two series of novel chrysin derivatives, linking 1,2,3-triazole and 1,2,4-triazole fragments in the $7-\mathrm{OH}$ position. The antiproliferative activity of the target compounds was evaluated on human gastric cancer (MGC-803), human hepatocellular carcinoma (BEL-7402), human liver cancer (HepG2), human cervical cancer (HeLa), human lung cancer (A549), and human gastric cancer (SGC-7901).

\section{Results and discussion}

\subsection{Chemistry}

Intermediate I was prepared by the nucleophilic substi- tution reaction of chrysin with propargyl bromide, and compounds $\mathbf{1 a} \sim \mathbf{1 h}$ were obtained by a click reaction of intermediate I with different substitutions of 1-(azidomethyl)benzene (yield: $31.3 \% \sim 41.2 \%$ ). Intermediate II was prepared by the nucleophilic substitution reaction of chrysin with ethyl bromoacetate, and intermediate III was generated from intermediate II and hydrazine hydrate. Compounds $\mathbf{2} \mathbf{a} \sim \mathbf{2} \mathbf{k}$ were products of the reaction of intermediate III with different substitutions of aniline at $82{ }^{\circ} \mathrm{C}$. The yields of $\mathbf{2} \mathbf{a} \sim \mathbf{2 k}$ were in the range of $10.3 \% \sim 25.5 \%$.

\subsection{Evaluation of the bioactivity}

As summarized in Table 1, compounds 1a, 1b, 1c, and 1g exhibited stronger antiproliferative activities against the MGC-803 cell line than the lead compound chrysin at 100 $\mu \mathrm{mol} / \mathrm{L}$, while compounds $\mathbf{1 e}, \mathbf{1 h}$, and $\mathbf{2 b}$ showed a considerably higher antiproliferative activity against the BEL-<smiles>[R]c1ccc(-n2cnnc2COc2cc(O)c3c(=O)cc(-c4ccccc4)oc3c2)cc1</smiles>

1a: $R=H, 1 b: R=4-F, 1 c: R=3-F, 1 d: R=2-F, 1 e: R=4-C l, 1 f: R=3-C l, 1 g: R=2-C l, 1 h: R=4-C_{3} ; 2 a: R=H, 2 b: R=4-F, 2 c: R=3-F, 2 d:$ $\mathrm{R}=2-\mathrm{F}, 2 \mathrm{e}: \mathrm{R}=4-\mathrm{Cl}, \mathbf{2 f}: \mathrm{R}=3-\mathrm{Cl}, \mathbf{2 g}: \mathrm{R}=2-\mathrm{Cl}, \mathbf{2 h}: \mathrm{R}=3-\mathrm{Br}, \mathbf{2 i}: \mathrm{R}=4-\mathrm{CH}_{3}$, 2j: $\mathrm{R}=3-\mathrm{CH}_{3}, \mathbf{2 j}: \mathrm{R}=4-\mathrm{OCH}_{3}$

Reagents and conditions: (a) propargyl bromide, $\mathrm{K}_{2} \mathrm{CO}_{3}, \mathrm{CH}_{3} \mathrm{COCH}_{3}, 57{ }^{\circ} \mathrm{C}$; (b) different substitutions of 1-(azidomethyl)benzene, CuSO $\mathrm{O}_{4}$. $5 \mathrm{H}_{2} \mathrm{O}$, sodium ascorbate, $t-\mathrm{BuOH} / \mathrm{H}_{2} \mathrm{O}(\mathrm{V}: \mathrm{V}=1: 1), 30{ }^{\circ} \mathrm{C}$; (c) ethyl bromoacetate, $\mathrm{K}_{2} \mathrm{CO}_{3}, \mathrm{CH}_{3} \mathrm{COCH}_{3}, 57{ }^{\circ} \mathrm{C}$; $(\mathrm{d}) \mathrm{N}_{2} \mathrm{H}_{4} \cdot \mathrm{H}_{2} \mathrm{O}, \mathrm{CH}_{3} \mathrm{CH}_{2} \mathrm{OH}$, $78{ }^{\circ} \mathrm{C}$; (e) DMFDMA, $\mathrm{CH}_{3} \mathrm{CN}$, different substitutions of aniline, $\mathrm{CH}_{3} \mathrm{COOH}, 82{ }^{\circ} \mathrm{C}$

Scheme 1 Synthetic routes of the target compounds 
Table 1 Antiproliferative activity of compounds ${ }^{a}$

\begin{tabular}{ccccccc}
\hline \multirow{2}{*}{ Compd. } & \multicolumn{7}{c}{ Growth inhibition/\% at $100 \mu \mathrm{mol} / \mathrm{L}$} & HeLa & A549 & SGC-7901 \\
\cline { 2 - 7 } 1a & MGC-803 & BEL-7402 & HepG-2 & H1.90 \pm 0.99 & $44.67 \pm 0.72$ & $19.31 \pm 0.65$ \\
$\mathbf{1 b}$ & $52.71 \pm 0.71$ & $44.01 \pm 0.77$ & $11.89 \pm 0.86$ & $24.42 \pm 1.01$ & $41.20 \pm 1.55$ & $47.68 \pm 1.36$ \\
$\mathbf{1 c}$ & $52.82 \pm 2.25$ & $3.31 \pm 0.07$ & $1.02 \pm 0.02$ & $16.81 \pm 0.17$ & $27.89 \pm 0.56$ & $7.30 \pm 0.14$ \\
$\mathbf{1 d}$ & $41.52 \pm 1.12$ & $31.44 \pm 0.88$ & $4.91 \pm 0.05$ & $24.40 \pm 0.26$ & $27.89 \pm 0.19$ & $12.78 \pm 0.18$ \\
$\mathbf{1 e}$ & $33.81 \pm 0.25$ & $98.18 \pm 1.35$ & $6.81 \pm 0.07$ & $6.32 \pm 0.02$ & $33.91 \pm 0.67$ & $12.64 \pm 0.13$ \\
$\mathbf{1 f}$ & $10.59 \pm 0.08$ & $12.80 \pm 0.14$ & $7.43 \pm 0.25$ & $16.70 \pm 0.09$ & $43.46 \pm 0.22$ & $20.98 \pm 0.15$ \\
$\mathbf{1 g}$ & $74.28 \pm 1.68$ & $17.44 \pm 0.12$ & $33.02 \pm 0.26$ & $35.07 \pm 0.18$ & $50.71 \pm 0.87$ & $45.64 \pm 0.88$ \\
$\mathbf{1 h}$ & $78.33 \pm 0.36$ & $97.60 \pm 0.98$ & $12.92 \pm 0.11$ & $4.11 \pm 0.17$ & $29.13 \pm 0.14$ & $6.44 \pm 0.12$ \\
$\mathbf{2 a}$ & $27.33 \pm 0.11$ & $57.24 \pm 0.76$ & $34.01 \pm 0.33$ & $27.30 \pm 0.05$ & $39.49 \pm 0.17$ & $51.71 \pm 1.00$ \\
$\mathbf{2 b}$ & $44.51 \pm 0.57$ & $78.26 \pm 1.77$ & $5.11 \pm 0.02$ & $26.81 \pm 0.18$ & $9.01 \pm 0.07$ & $6.81 \pm 0.05$ \\
$\mathbf{2 c}$ & $10.72 \pm 0.12$ & $70.70 \pm 1.02$ & $4.52 \pm 0.12$ & $26.42 \pm 0.26$ & $15.01 \pm 0.07$ & $8.22 \pm 0.12$ \\
$\mathbf{2 d}$ & $34.62 \pm 0.23$ & $15.49 \pm 0.11$ & $88.40 \pm 1.66$ & $29.33 \pm 0.20$ & $44.11 \pm 0.31$ & $49.22 \pm 0.32$ \\
$\mathbf{2 e}$ & $25.21 \pm 0.21$ & $32.30 \pm 0.33$ & $7.50 \pm 0.08$ & $35.41 \pm 0.37$ & $31.11 \pm 0.33$ & $19.70 \pm 0.26$ \\
$\mathbf{2 f}$ & $22.62 \pm 0.24$ & $44.33 \pm 0.08$ & $15.62 \pm 0.12$ & $40.92 \pm 0.40$ & $37.80 \pm 0.08$ & $37.71 \pm 0.37$ \\
$\mathbf{2 g}$ & $31.71 \pm 0.15$ & $19.01 \pm 0.12$ & $31.02 \pm 0.24$ & $20.10 \pm 0.20$ & $27.90 \pm 0.13$ & $18.41 \pm 0.10$ \\
$\mathbf{2 h}$ & $16.80 \pm 0.10$ & $35.71 \pm 0.11$ & $38.79 \pm 0.31$ & $46.50 \pm 0.40$ & $43.01 \pm 0.23$ & $47.83 \pm 0.32$ \\
$\mathbf{2 i}$ & $42.11 \pm 0.34$ & $49.81 \pm 0.37$ & $38.82 \pm 0.21$ & $35.51 \pm 0.13$ & $30.11 \pm 0.13$ & $51.09 \pm 0.05$ \\
$\mathbf{2 j}$ & $36.89 \pm 0.33$ & $57.64 \pm 0.32$ & $51.60 \pm 0.22$ & $30.49 \pm 0.61$ & $46.02 \pm 0.64$ & $47.11 \pm 0.69$ \\
$\mathbf{2 k}$ & $21.73 \pm 0.27$ & $29.44 \pm 0.04$ & $58.11 \pm 0.97$ & $38.22 \pm 0.22$ & $44.52 \pm 0.12$ & $36.54 \pm 0.20$ \\
Chrysin & $50.89 \pm 0.50$ & $73.02 \pm 0.23$ & $39.33 \pm 0.22$ & $62.89 \pm 0.33$ & $8.80 \pm 0.04$ & $16.30 \pm 0.13$ \\
\hline
\end{tabular}

${ }^{a}$ Growth inhibitions are presented as the mean \pm SD (standard error of the mean) from three separated experiment.

7402 cell line. However, most compounds lost their antiproliferative activity at lower concentrations and only four compounds had an $\mathrm{IC}_{50}$ value $(<100 \mu \mathrm{mol} / \mathrm{L})$ lower than that of chrysin against the MGC-803 cell line.

As listed in Table 2, only compounds 1a, 1b, 1c and $\mathbf{1 g}$ exhibited higher antiproliferative activities, indicating that the antiproliferative activity induced by introducing 1,2,3triazole in the 7-hydroxyl group of chrysin was better than that induced by the introduction of 1,2,4-triazole. Among them, compounds 1c and 1g exhibited significant antiproliferative potency against the MGC-803 cell line with $\mathrm{IC}_{50}$ values of $(18.4 \pm 0.14)$ and $(5.92 \pm 0.02) \mu \mathrm{mol} / \mathrm{L}$, respectively, which were even better than that of 5-fluorouracil (5-FU). Compound 1g, with a chlorine atom introduced at the ortho position of the benzene ring, had the strongest antiproliferative activity among all target compounds, and improved potency up to two digits $\mu \mathrm{mol} / \mathrm{L}$ compared with that of chrysin. It is possible that the chlorine atom acts as a hydrogen bond acceptor and binds to some key proteins in- volved in cancer cell metabolism, inhibiting their expression. Compound $\mathbf{1 g}$ was 5.2-folds more active than

Table $2 \mathrm{IC}_{50}$ values $(\mu \mathrm{mol} / \mathrm{L})$ of several active compounds

\begin{tabular}{cc}
\hline Compd. & $\mathrm{IC}_{50}$ against $\mathrm{MGC}-803$ cell lines $/\left(\mu \mathrm{mol} \cdot \mathrm{L}^{-1}\right)$ \\
\hline $\mathbf{1 a}$ & $26.79 \pm 0.03$ \\
$\mathbf{1 b}$ & $95.58 \pm 0.07$ \\
$\mathbf{1 c}$ & $18.40 \pm 0.14$ \\
$\mathbf{1 g}$ & $5.92 \pm 0.02$ \\
Chrysin & $>100$ \\
5 -FU & $30.52 \pm 0.36$
\end{tabular}

$\mathrm{IC}_{50}$ : concentration that inhibits $50 \%$ of cell growth. The values are presented as the mean $\pm \mathrm{SD}$ (standard error of the mean) from three separated experiments.
5-FU, which demonstrated that it may be a valuable candidate for further studies.

\section{Conclusions}

In summary, 19 novel chrysin derivatives were synthesized and their chemical structures were characterized via ${ }^{1} \mathrm{H}$ NMR, ${ }^{13} \mathrm{C}$ NMR, IR and mass spectra. Biological assays revealed that the introduction of 1,2,3-triazole fragments in the 7-hydroxyl group of chrysin could improve its antiproliferative activity against MGC-803 cell lines. These results are useful for research on more potent novel antiproliferative compounds and further studies are ongoing.

\section{Experimental section}

All reactions were monitored by thin-layer chromatography (TLC) performed on silica gel plates. IR spectra were recorded (in $\mathrm{KBr}$ ) on an IR Prestige-21. ${ }^{1} \mathrm{H}$ NMR and ${ }^{13} \mathrm{C}$ NMR spectra were recorded on an AV-300 spectrometers (Bruker BioSpin, Switzerland) at room temperature, tetramethylsilane (TMS) was used as the internal standard. Mass spectra were measured using a matrix-assisted laser desorption/ionization (MALDI)-time of flight (TOF)/TOF mass spectrometer (Bruker Daltonik, Germany). The major chemicals were purchased from Aldrich Chemical Corporation (Milwaukee, WI). High-resolution mass spectra were recorded using a Thermo Scientific LTQ Orbitrap XL in the electrospray ionisation (ESI) mode. All other chemicals were analytical grade.

3-[4,5-Dimethylthiazol-2-yl]-2,5-diphenyl-tetrazolium bromide (MTT) was purchased from Sigma Chemical Co. (St. Louis, MO). All cells were initially purchased from 
American Type Culture Collection (ATCC, Manassas, VA, USA). Dulbecco's modified Eagle's medium (DMEM), RPMI-1640 media, and foetal bovine serum (FBS) were provided from Gibco Company (Grand Island, NY, USA). The cells were maintained in RPMI-1640 or DMEM, supplemented with $10 \% \mathrm{FBS}, 100 \mathrm{mg} / \mathrm{mL}$ streptomycin and $100 \mathrm{IU} / \mathrm{mL}$ penicillin (Grand Island, NY, USA), and at $37{ }^{\circ} \mathrm{C}$ in a humidified atmosphere containing $5 \% \mathrm{CO}_{2}$.

4.1 General procedure for the syntheses of intermediates I, II, and III

5-Hydroxy-2-phenyl-7-(prop-2-ynyloxy)-4H-chromen4-one (I) and 2-(5-hydroxy-4-oxo-2-phenyl-4H-chromen7-yloxy)acetohydrazide (III) were synthesized as per the protocol described in a previous study. ${ }^{[16,17]}$

Chrysin (1.27 g, $5.0 \mathrm{mmol}), \mathrm{K}_{2} \mathrm{CO}_{3}(1.00 \mathrm{~g}, 7.5 \mathrm{mmol})$ and ethyl bromoacetate $(1.00 \mathrm{~g}, 6.0 \mathrm{mmol})$ were added to $\mathrm{CH}_{3} \mathrm{COCH}_{3}(50 \mathrm{~mL})$. The mixture was stirred at $57{ }^{\circ} \mathrm{C}$ for $2 \mathrm{~h}$. Then, the solvent was evaporated in vacuo, water was added $(20 \mathrm{~mL})$, the mixture was filtered, and the residue was washed with water to obtain ethyl 2-(5-hydroxy-4oxo-2-phenyl-4H-chromen-7-yloxy)acetate (II): ${ }^{[18]}{ }^{1} \mathrm{H}$ NMR $\left(\mathrm{CDCl}_{3}, 300 \mathrm{MHz}\right) \delta: 12.83(\mathrm{~s}, 1 \mathrm{H}), 8.11(\mathrm{dd}, J=$ $7.9,1.5 \mathrm{~Hz}, 2 \mathrm{H}), 7.68 \sim 7.53(\mathrm{~m}, 3 \mathrm{H}), 7.08(\mathrm{~s}, 1 \mathrm{H}), 6.87(\mathrm{~d}$, $J=2.2 \mathrm{~Hz}, 1 \mathrm{H}), 6.44(\mathrm{~d}, J=2.2 \mathrm{~Hz}, 1 \mathrm{H}), 4.97(\mathrm{~s}, 2 \mathrm{H}), 4.19$ (q, $J=7.1 \mathrm{~Hz}, 2 \mathrm{H}), 1.23(\mathrm{t}, J=7.1 \mathrm{~Hz}, 3 \mathrm{H})$.

4.2 General procedure for the syntheses of compounds $1 \mathrm{a} \sim 1 \mathrm{~h}$

A mixture of compound I (146 mg, $0.5 \mathrm{mmol})$, different substitutions of 1 -(azidomethyl)benzene $(0.5 \mathrm{mmol})$, $\mathrm{CuSO}_{4} \cdot 5 \mathrm{H}_{2} \mathrm{O}(10 \mathrm{mg}, 0.05 \mathrm{mmol})$ and sodium ascorbate $(17 \mathrm{mg}, 0.1 \mathrm{mmol})$ were added to $t-\mathrm{BuOH} / \mathrm{H}_{2} \mathrm{O}(V: V=$ $1: 1,30 \mathrm{~mL}$ ) and stirred at $30{ }^{\circ} \mathrm{C}$ for $12 \mathrm{~h}$. After confirming the reaction progress by TLC, $t$ - $\mathrm{BuOH}$ was evaporated in vacuo. Then, the mixture was extracted with dichloromethane $(10 \mathrm{~mL} \times 3)$ and washed with saline $(10$ $\mathrm{mL})$. Finally, the mixture was purified using silica gel column chromatography and eluted with dichloromethane/methanol $(V: V=100: 1)$ to obtain the target compounds $\mathbf{1 a} \sim \mathbf{1 h}$.

5-Hydroxy-2-phenyl-7-((1-phenyl-1H-1,2,3-triazol-4-' yl)methoxy)-4H-chromen-4-one (1a): Pale yellow solid, yield $41.2 \%$. m.p. $186 \sim 187{ }^{\circ} \mathrm{C}$; ${ }^{1} \mathrm{H}$ NMR $\left(\mathrm{CDCl}_{3}, 300\right.$ $\mathrm{MHz}) \delta: 12.72(\mathrm{~s}, 1 \mathrm{H}, \mathrm{OH}), 7.88(\mathrm{~d}, J=5.9 \mathrm{~Hz}, 2 \mathrm{H}, \mathrm{ArH})$, $7.57 \sim 7.52(\mathrm{~m}, 4 \mathrm{H}, \mathrm{ArH}), 7.40 \sim 7.37(\mathrm{~m}, 3 \mathrm{H}, \mathrm{ArH})$, $7.31 \sim 7.26(\mathrm{~m}, 2 \mathrm{H}, \mathrm{ArH}), 6.67(\mathrm{~s}, 1 \mathrm{H}, \mathrm{ArH}), 6.61$ (d, $J=$ $2.0 \mathrm{~Hz}, 1 \mathrm{H}, \mathrm{ArH}), 6.41$ (d, $J=2.0 \mathrm{~Hz}, 1 \mathrm{H}, \mathrm{ArH}), 5.55$ (s, $\left.2 \mathrm{H}, \mathrm{CH}_{2}\right), 5.26\left(\mathrm{~s}, 2 \mathrm{H}, \mathrm{CH}_{2}\right) ;{ }^{13} \mathrm{C} \mathrm{NMR}\left(\mathrm{CDCl}_{3}, 75 \mathrm{MHz}\right)$ $\delta: 182.50,164.13,164.03,162.15,157.77,134.28,133.18$, $131.92,131.21,129.22$ (2C), 129.11 (2C), 128.95, 128.20 (2C), 126.34 (2C), 122.88, 106.08, 105.92, 99.06, 93.27, 62.42, 54.39; IR (KBr) v: 3138, 1660, 1612, 1498, 1446, 1350, $1165 \mathrm{~cm}^{-1}$; MS-MALDI $m / z: 426\left[\mathrm{M}+\mathrm{H}^{+}\right]$; HRMS (ESI) calcd for $\mathrm{C}_{25} \mathrm{H}_{20} \mathrm{~N}_{3} \mathrm{O}_{4}\left[\mathrm{M}+\mathrm{H}^{+}\right]$426.1448, found 426.1443 .

7-((1-(4-Fluorophenyl)-1H-1,2,3-triazol-4-yl)methoxy)5-hydroxy-2-phenyl-4H-chromen-4-one (1b): Pale yellow solid, yield 39.5\%. m.p. 171 173 ${ }^{\circ} \mathrm{C} ;{ }^{1} \mathrm{H} \mathrm{NMR}\left(\mathrm{CDCl}_{3}\right.$, $300 \mathrm{MHz}) \delta: 12.75(\mathrm{~s}, 1 \mathrm{H}, \mathrm{OH}), 7.91(\mathrm{dd}, J=7.7,1.9 \mathrm{~Hz}$, $2 \mathrm{H}, \mathrm{ArH}), 7.59 \sim 7.52(\mathrm{~m}, 4 \mathrm{H}, \mathrm{ArH}), 7.34 \sim 7.29(\mathrm{~m}, 2 \mathrm{H}$, ArH), $7.13 \sim 7.07$ (m, 2H, ArH), 6.70 (s, 1H, ArH), 6.63 (d, $J=2.2 \mathrm{~Hz}, 1 \mathrm{H}, \mathrm{ArH}), 6.44(\mathrm{~d}, J=2.2 \mathrm{~Hz}, 1 \mathrm{H}, \mathrm{ArH})$, $5.55\left(\mathrm{~s}, 2 \mathrm{H}, \mathrm{CH}_{2}\right), 5.30\left(\mathrm{~s}, 2 \mathrm{H}, \mathrm{CH}_{2}\right) ;{ }^{13} \mathrm{C} \mathrm{NMR}\left(\mathrm{CDCl}_{3}, 75\right.$ MHz) $\delta: 182.50,164.16,163.99,162.90(\mathrm{~d}, J=151.5 \mathrm{~Hz})$, $162.16,157.77,143.60,137.79,131.94,131.19,130.10(\mathrm{~d}$, $J=151.5 \mathrm{~Hz}, 2 \mathrm{C}), 129.12$ (2C), 126.34 (2C), 122.74, $116.26(\mathrm{~d}, J=21.8 \mathrm{~Hz}, 2 \mathrm{C}), 106.09,105.92,99.02,93.27$, 62.38, 53.62; IR (KBr) v: 3136, 1662, 1614, 1502, 1452, 1348, $1165 \mathrm{~cm}^{-1}$; MS-MALDI $m / z: 444\left[\mathrm{M}+\mathrm{H}^{+}\right.$]; HRMS (ESI) calcd for $\mathrm{C}_{25} \mathrm{H}_{20} \mathrm{~N}_{3} \mathrm{O}_{4}\left[\mathrm{M}+\mathrm{H}^{+}\right]$444.1354, found 444.1354.

7-((1-(3-Fluorophenyl)-1H-1,2,3-triazol-4-yl)methoxy)5-hydroxy-2-phenyl-4H-chromen-4-one (1c): Pale yellow solid, yield 33.5\%. m.p. $175 \sim 177{ }^{\circ} \mathrm{C} ;{ }^{1} \mathrm{H} \mathrm{NMR}\left(\mathrm{CDCl}_{3}\right.$, $300 \mathrm{MHz}) \delta: 12.75(\mathrm{~s}, 1 \mathrm{H}, \mathrm{OH}), 7.91(\mathrm{dd}, J=7.7,1.8 \mathrm{~Hz}$, $2 \mathrm{H}, \mathrm{ArH}), 7.63 \sim 7.51(\mathrm{~m}, 4 \mathrm{H}, \mathrm{ArH}), 7.38(\mathrm{dd}, J=13.8,7.9$ $\mathrm{Hz}, 1 \mathrm{H}, \mathrm{ArH}), 7.11 \sim 7.06$ (m, 2H, ArH), 7.00 (d, $J=9.2$ $\mathrm{Hz}, 1 \mathrm{H}, \mathrm{ArH}), 6.69$ (s, 1H, ArH), 6.64 (d, $J=2.2 \mathrm{~Hz}, 1 \mathrm{H}$, $\mathrm{ArH}), 6.44(\mathrm{~d}, J=2.2 \mathrm{~Hz}, 1 \mathrm{H}, \mathrm{ArH}), 5.57\left(\mathrm{~s}, 2 \mathrm{H}, \mathrm{CH}_{2}\right)$, $5.31\left(\mathrm{~s}, 2 \mathrm{H}, \mathrm{CH}_{2}\right) ;{ }^{13} \mathrm{C} \mathrm{NMR}\left(\mathrm{CDCl}_{3}, 75 \mathrm{MHz}\right) \delta: 182.51$, $168.44(\mathrm{~d}, J=132.0 \mathrm{~Hz}), 164.16,163.97,162.15,157.76$, $143.69,136.64$ (d, $J=7.5 \mathrm{~Hz}), 131.95,131.18,130.91$ (d, $J=8.3 \mathrm{~Hz}$ ), 129.13 (2C), 126.35 (2C), 123.66 (d, $J=3.0$ $\mathrm{Hz}), 122.96,116.00(\mathrm{~d}, J=21.0 \mathrm{~Hz}), 115.13$ (d, $J=21.8$ $\mathrm{Hz}), 114.98,105.91,99.03$, 93.26, 62.37, 53.71; IR (KBr) $v: 3165,1660,1612,1502,1450,1359,1168 \mathrm{~cm}^{-1}$. MS-MALDI $m / z: 444\left[\mathrm{M}+\mathrm{H}^{+}\right]$; HRMS (ESI) calcd for $\mathrm{C}_{25} \mathrm{H}_{19} \mathrm{FN}_{3} \mathrm{O}_{4}\left[\mathrm{M}+\mathrm{H}^{+}\right]$444.1354, found 444.1354.

7-((1-(2-Fluorophenyl)-1H-1,2,3-triazol-4-yl)methoxy)5-hydroxy-2-phenyl-4H-chromen-4-one (1d): Pale yellow solid, yield 31.3\%. m.p. 201 203 ${ }^{\circ} \mathrm{C} ;{ }^{1} \mathrm{H}$ NMR $\left(\mathrm{CDCl}_{3}\right.$, $300 \mathrm{MHz}) \delta: 12.75(\mathrm{~s}, 1 \mathrm{H}, \mathrm{OH}), 7.91(\mathrm{dd}, J=7.6,1.9 \mathrm{~Hz}$, 2H, ArH), 7.70 (s, 1H, ArH), $7.57 \sim 7.54$ (m, 3H, ArH), $7.32 \sim 7.34(\mathrm{~m}, 2 \mathrm{H}, \mathrm{ArH}), 7.21 \sim 7.12(\mathrm{~m}, 2 \mathrm{H}, \mathrm{ArH}), 6.70$ (s, 1H, ArH), 6.65 (d, $J=2.2 \mathrm{~Hz}, 1 \mathrm{H}, \mathrm{ArH}), 6.45$ (d, $J=$ $2.2 \mathrm{~Hz}, 1 \mathrm{H}, \mathrm{ArH}), 5.64\left(\mathrm{~s}, 2 \mathrm{H}, \mathrm{CH}_{2}\right), 5.28\left(\mathrm{~s}, 2 \mathrm{H}, \mathrm{CH}_{2}\right)$; ${ }^{13} \mathrm{C} \mathrm{NMR}\left(\mathrm{CDCl}_{3}, 75 \mathrm{MHz}\right) \delta: 182.53,164.15,164.03$, $162.14,158.86$ (d, $J=165.0 \mathrm{~Hz}), 143.44,131.94,131.21$ $(\mathrm{d}, J=0.8 \mathrm{~Hz}), 131.10,130.75(\mathrm{~d}, J=3.0 \mathrm{~Hz}), 129.13$ (2C), 126.35 (2C), 124.97, 124.92, 123.14, 123.12, 118.83 $(\mathrm{d}, J=19.5 \mathrm{~Hz}), 115.95(\mathrm{~d}, J=21.0 \mathrm{~Hz}), 106.80,99.05$, 93.27, 62.36, 50.92; IR (KBr) v: 3134, 1670, 1614, 1496, 1452, 1350, $1161 \mathrm{~cm}^{-1}$; MS-MALDI $\mathrm{m} / z: 444\left[\mathrm{M}+\mathrm{H}^{+}\right]$; HRMS (ESI) calcd for $\mathrm{C}_{25} \mathrm{H}_{20} \mathrm{~N}_{3} \mathrm{O}_{4}\left[\mathrm{M}+\mathrm{H}^{+}\right]$444.1354, found 444.1353 .

7-((1-(4-Chlorophenyl)-1H-1,2,3-triazol-4-yl)methoxy)5-hydroxy-2-phenyl-4H-chromen-4-one (1e): Pale yellow solid, yield 37.2\%. m.p. 172 173 ${ }^{\circ} \mathrm{C} ;{ }^{1} \mathrm{H} \mathrm{NMR}\left(\mathrm{CDCl}_{3}\right.$, $300 \mathrm{MHz}) \delta: 12.74(\mathrm{~s}, 1 \mathrm{H}, \mathrm{OH}), 7.92 \sim 7.89(\mathrm{~m}, 2 \mathrm{H}, \mathrm{ArH})$, $7.60 \sim 7.54(\mathrm{~m}, 4 \mathrm{H}, \mathrm{ArH}), 7.40 \sim 7.37(\mathrm{~m}, 2 \mathrm{H}, \mathrm{ArH})$, $7.28 \sim 7.24(\mathrm{~m}, 2 \mathrm{H}, \mathrm{ArH}), 6.69(\mathrm{~s}, 1 \mathrm{H}, \mathrm{ArH}), 6.63(\mathrm{~d}, J=$ $2.2 \mathrm{~Hz}, 1 \mathrm{H}, \mathrm{ArH}), 6.43(\mathrm{~d}, J=2.2 \mathrm{~Hz}, 1 \mathrm{H}, \mathrm{ArH}), 5.54(\mathrm{~s}$, $\left.2 \mathrm{H}, \mathrm{CH}_{2}\right), 5.30\left(\mathrm{~s}, 2 \mathrm{H}, \mathrm{CH}_{2}\right) ;{ }^{13} \mathrm{C} \mathrm{NMR}\left(\mathrm{CDCl}_{3}, 75 \mathrm{MHz}\right)$ $\delta: 182.51,164.17,163.98,162.17,157.77,143.69,135.04$, 
$132.77,131.95,131.20,129.51$ (2C), 129.45 (2C), 129.13 (2C), 126.35 (2C), 122.80, 106.10, 105.93, 99.02, 93.28, 62.37, 53.62; IR (KBr) v: 3128, 1664, 1612, 1496, 1450, 1346, $1161 \mathrm{~cm}^{-1}$; MS-MALDI $m / z: 460\left[\mathrm{M}+\mathrm{H}^{+}\right]$; HRMS (ESI) calcd for $\mathrm{C}_{25} \mathrm{H}_{19} \mathrm{ClN}_{3} \mathrm{O}_{4}\left[\mathrm{M}+\mathrm{H}^{+}\right]$460.1058, found 460.1056.

7-((1-(3-Chlorophenyl)-1H-1,2,3-triazol-4-yl)methoxy)5-hydroxy-2-phenyl-4H-chromen-4-one (1f): Pale yellow solid, yield 35.1\%. m.p. 152 153 ${ }^{\circ} \mathrm{C}$; ${ }^{1} \mathrm{H}$ NMR $\left(\mathrm{CDCl}_{3}\right.$, $300 \mathrm{MHz}) \delta: 12.74(\mathrm{~s}, 1 \mathrm{H}, \mathrm{OH}), 7.91 \sim 7.88(\mathrm{~m}, 2 \mathrm{H}, \mathrm{ArH})$, $7.63 \sim 7.54(\mathrm{~m}, 4 \mathrm{H}, \mathrm{ArH}), 7.45 \sim 7.29$ (m, 3H, ArH), 7.19 $(\mathrm{d}, J=4.7 \mathrm{~Hz}, 1 \mathrm{H}, \mathrm{ArH}), 6.70(\mathrm{~s}, 1 \mathrm{H}, \mathrm{ArH}), 6.63(\mathrm{~d}, J=$ $2.2 \mathrm{~Hz}, 1 \mathrm{H}, \mathrm{ArH}), 6.44$ (d, $J=2.1 \mathrm{~Hz}, 1 \mathrm{H}, \mathrm{ArH}), 5.55$ (s, $\left.2 \mathrm{H}, \mathrm{CH}_{2}\right), 5.29\left(\mathrm{~s}, 2 \mathrm{H}, \mathrm{CH}_{2}\right) ;{ }^{13} \mathrm{C} \mathrm{NMR}\left(\mathrm{CDCl}_{3}, 75 \mathrm{MHz}\right)$ $\delta: 182.52,164.17,163.96,162.14,157.77,143.73,136.20$, $135.11,131.96,131.16,130.54,129.18,129.13$ (2C), $128.20,126.35$ (2C), 126.20, 122.96, 106.09, 105.90, 99.05, 93.26, 62.35, 53.65; IR (KBr) v: 3072, 1666, 1612, $1498,1452,1354,1165 \mathrm{~cm}^{-1}$; MS-MALDI $\mathrm{m} / \mathrm{z}: 460[\mathrm{M}+$ $\mathrm{H}^{+}$]; HRMS (ESI) calcd for $\mathrm{C}_{25} \mathrm{H}_{19} \mathrm{ClN}_{3} \mathrm{O}_{4}\left[\mathrm{M}+\mathrm{H}^{+}\right]$ 460.1059, found 460.1060 .

7-((1-(2-Chlorophenyl)-1H-1,2,3-triazol-4-yl)methoxy)5-hydroxy-2-phenyl-4H-chromen-4-one (1g): Pale yellow solid, yield 31.7\%. m.p. $146 \sim 147{ }^{\circ} \mathrm{C}$; ${ }^{1} \mathrm{H} \mathrm{NMR}\left(\mathrm{CDCl}_{3}\right.$, $300 \mathrm{MHz}) \delta: 12.75$ (s, 1H, OH), $7.92 \sim 7.89$ (m, 2H, ArH), $7.71(\mathrm{~s}, 1 \mathrm{H}, \mathrm{ArH}), 7.56 \sim 7.45(\mathrm{~m}, 5 \mathrm{H}, \mathrm{ArH}), 7.37 \sim 7.30$ $(\mathrm{m}, 2 \mathrm{H}, \mathrm{ArH}), 6.70 \sim 6.64(\mathrm{~m}, 2 \mathrm{H}, \mathrm{ArH}), 6.45(\mathrm{~d}, J=2.1$ $\mathrm{Hz}, 1 \mathrm{H}, \mathrm{ArH}), 5.71\left(\mathrm{~s}, 2 \mathrm{H}, \mathrm{CH}_{2}\right), 5.29\left(\mathrm{~s}, 2 \mathrm{H}, \mathrm{CH}_{2}\right) ;{ }^{13} \mathrm{C}$ $\mathrm{NMR}\left(\mathrm{CDCl}_{3}, 75 \mathrm{MHz}\right) \delta: 182.52,164.15,164.02,162.13$, $157.76,143.32,133.61,132.13,131.94,131.19,130.57$, $130.48,130.04,129.13$ (2C), 127.70, 126.35 (2C), 123.28, 106.08, 105.91, 99.06, 93.30, 62.38, 51.63; IR (KBr) $v$ : $3143,1660,1614,1498,1444,1377,1159 \mathrm{~cm}^{-1}$; MSMALDI $m / z: 460\left[\mathrm{M}+\mathrm{H}^{+}\right]$; HRMS (ESI) calcd for $\mathrm{C}_{25} \mathrm{H}_{19^{-}}$ $\mathrm{ClN}_{3} \mathrm{O}_{4}\left[\mathrm{M}+\mathrm{H}^{+}\right]$460.1059, found 460.1055.

5-Hydroxy-2-phenyl-7-((1-(p-tolyl)-1H-1,2,3-triazol-4yl)methoxy)-4H-chromen-4-one (1h): Pale yellow solid, yield 37.8\%. m.p. 166 168 ${ }^{\circ} \mathrm{C}$; ${ }^{1} \mathrm{H}$ NMR $\left(\mathrm{CDCl}_{3}, 300\right.$ $\mathrm{MHz}) \delta: 12.82(\mathrm{~s}, 1 \mathrm{H}, \mathrm{OH}), 8.32(\mathrm{~s}, 1 \mathrm{H}, \mathrm{ArH}), 8.10 \sim 8.08$ $(\mathrm{m}, 2 \mathrm{H}, \mathrm{ArH}), 7.64 \sim 7.56(\mathrm{~m}, 3 \mathrm{H}, \mathrm{ArH}), 7.21$ (dd, $J=$ 20.2, 8.0 Hz, 4H, ArH), 7.05 (s, 1H, ArH), 6.94 (d, $J=2.1$ $\mathrm{Hz}, 1 \mathrm{H}, \mathrm{ArH}), 6.49$ (d, J=2.1 Hz, 1H, ArH), 5.57 (s, 2H, $\mathrm{ArH}), 5.28(\mathrm{~s}, 2 \mathrm{H}, \mathrm{ArH}), 2.27\left(\mathrm{~s}, 3 \mathrm{H}, \mathrm{CH}_{3}\right) ;{ }^{13} \mathrm{C} \mathrm{NMR}$ $\left(\mathrm{CDCl}_{3}, 75 \mathrm{MHz}\right) \delta: 182.31,163.93,161.97,157.59$, $143.30,138.77,131.79,131.16,131.03,129.74$ (2C), 128.97 (2C), 128.72, 128.13 (2C), 126.18 (2C), 122.76, $105.90,105.72,98.92,93.11,62.29,54.04,21.07$; IR (KBr) v: 3120, 1656, 1620, 1496, 1446, 1377, $1157 \mathrm{~cm}^{-1}$; MS-MALDI $\mathrm{m} / \mathrm{z}: 440\left[\mathrm{M}+\mathrm{H}^{+}\right]$; HRMS (ESI) calcd for $\mathrm{C}_{26} \mathrm{H}_{22} \mathrm{~N}_{3} \mathrm{O}_{4}\left[\mathrm{M}+\mathrm{H}^{+}\right]$440.1605, found 440.1602.

\subsection{General procedure for the syntheses of com- pounds $2 \mathrm{a} \sim 2 \mathrm{k}$}

Compound III (652.0 mg, $2.0 \mathrm{mmol})$ and $N, N$-dimethylformamide dimethyl acetal (DMFDMA, $260.0 \mathrm{mg}, 2.2$ mmol) were added to $\mathrm{CH}_{3} \mathrm{CN}(30 \mathrm{~mL})$. The mixture was stirred at $50{ }^{\circ} \mathrm{C}$ for $2 \mathrm{~h}$. Then, different substitutions of aniline $(3.0 \mathrm{mmol})$ and $\mathrm{CH}_{3} \mathrm{COOH}(0.72 \mathrm{~g}, 12 \mathrm{mmol})$ were added, and the mixture was stirred at $82{ }^{\circ} \mathrm{C}$ for $12 \mathrm{~h}$. After confirming the reaction progress by TLC, the solvent was evaporated in vacuo. The mixture was then purified using silica gel column chromatography and eluted using a gradient of dichloromethane/methanol $(V: V=120: 1)$ to obtain the target compounds $\mathbf{2} \mathbf{a} \sim \mathbf{2} \mathbf{k}$.

5-Hydroxy-2-phenyl-7-((4-phenyl-4H-1,2,4-triazol-3yl)methoxy)-4H-chromen-4-one (2a): Pale yellow solid, Yield 23.5\%. m.p. $188 \sim 189{ }^{\circ} \mathrm{C} ;{ }^{1} \mathrm{H}$ NMR (DMSO- $d_{6}$, $300 \mathrm{MHz}) \delta: 12.80(\mathrm{~s}, 1 \mathrm{H}, \mathrm{OH}), 8.92(\mathrm{~s}, 1 \mathrm{H}$, Triazole-H), 8.07 (d, $J=7.0 \mathrm{~Hz}, 2 \mathrm{H}, \mathrm{ArH}), 7.78 \sim 7.35$ (m, 8H, ArH), 7.03 (s, 1H, ArH), 6.85 (d, $J=1.5 \mathrm{~Hz}, 1 \mathrm{H}, \mathrm{ArH}), 6.40$ (d, $J=1.5 \mathrm{~Hz}, 1 \mathrm{H}, \mathrm{ArH}), 5.38\left(\mathrm{~s}, 2 \mathrm{H}, \mathrm{CH}_{2}\right) ;{ }^{13} \mathrm{C} \mathrm{NMR}$ (DMSO- $\left.d_{6}, 75 \mathrm{MHz}\right) \delta$ : 187.89, 182.58, 164.07, 163.62, $161.60,157.58,149.17,145.88,134.02,132.67,130.98$, $130.27,129.85,129.63,126.92,125.77,105.91,105.85$, 99.17, 94.17, 60.48; IR (KBr) v: 3089, 1666, 1622, 1502, 1450, 1550, 1338, $1153 \mathrm{~cm}^{-1}$; MS-MALDI $\mathrm{m} / \mathrm{z}: 412$ $\left[\mathrm{M}+\mathrm{H}^{+}\right]$; HRMS (ESI) calcd for $\mathrm{C}_{26} \mathrm{H}_{22} \mathrm{~N}_{3} \mathrm{O}_{4}\left[\mathrm{M}+\mathrm{H}^{+}\right]$ 412.1292, found 412.1291.

7-((4-(4-Fluorophenyl)-4H-1,2,4-triazol-3-yl)methoxy)5-hydroxy-2-phenyl-4H-chromen-4-one (2b): Pale yellow solid, yield $24.5 \%$. m.p. $177 \sim 179{ }^{\circ} \mathrm{C} ;{ }^{1} \mathrm{H}$ NMR $\left(\mathrm{CDCl}_{3}\right.$, $300 \mathrm{MHz}) \delta: 12.76(\mathrm{~s}, 1 \mathrm{H}, \mathrm{OH}), 8.35$ (s, 1H, Triazole-H), $7.91(\mathrm{~d}, J=6.0 \mathrm{~Hz}, 2 \mathrm{H}, \mathrm{ArH}), 7.57 \sim 7.42(\mathrm{~m}, 5 \mathrm{H}, \mathrm{ArH})$, 7.27 (d, J=6.0 Hz, 2H, ArH), 6.70 (s, 2H, ArH), 6.35 (s, $1 \mathrm{H}, \mathrm{ArH}), 5.29\left(\mathrm{~d}, J=15.5 \mathrm{~Hz}, 2 \mathrm{H}, \mathrm{CH}_{2}\right) ;{ }^{13} \mathrm{C} \mathrm{NMR}$ $\left(\mathrm{CDCl}_{3}, 75 \mathrm{MHz}\right) \delta$ : 182.49, 164.32, 162.92, 162.25, $159.84(\mathrm{~d}, J=135.8 \mathrm{~Hz}), 157.76,149.40,144.83,132.04$, $131.06,130.75,129.29$ (d, $J=3.0 \mathrm{~Hz}), 129.14$ (2C), 127.56 (d, $J=9.0 \mathrm{~Hz}, 2 \mathrm{C}), 126.38$ (2C), 117.20 (d, $J=23.3$ $\mathrm{Hz}, 2 \mathrm{C}), 106.51,106.00,99.04,93.11,59.63$; IR (KBr) v: 3068, 1654, 1614, 1512, 1496, 1450, 1338, $1155 \mathrm{~cm}^{-1}$; MS-MALDI $m / z$ : $430\left[\mathrm{M}+\mathrm{H}^{+}\right.$]; HRMS (ESI) calcd for $\mathrm{C}_{24} \mathrm{H}_{17} \mathrm{FN}_{3} \mathrm{O}_{4}\left[\mathrm{M}+\mathrm{H}^{+}\right]$430.1198, found 430.1197.

7-((4-(3-Fluorophenyl)-4H-1,2,4-triazol-3-yl)methoxy)5-hydroxy-2-phenyl-4H-chromen-4-one (2c): Pale yellow solid, yield $21.5 \%$. m.p. $174 \sim 176{ }^{\circ} \mathrm{C} ;{ }^{1} \mathrm{H} \mathrm{NMR}\left(\mathrm{CDCl}_{3}\right.$, $300 \mathrm{MHz}) \delta: 12.76(\mathrm{~s}, 1 \mathrm{H}, \mathrm{OH}), 8.39(\mathrm{~s}, 1 \mathrm{H}$, Triazole-H), $7.93 \sim 7.90(\mathrm{~m}, 2 \mathrm{H}, \mathrm{ArH}), 7.55 \sim 7.53(\mathrm{~m}, 3 \mathrm{H}, \mathrm{ArH})$, $7.28 \sim 7.25(\mathrm{~m}, 4 \mathrm{H}, \mathrm{ArH}), 6.76 \sim 6.68(\mathrm{~m}, 2 \mathrm{H}, \mathrm{ArH}), 6.37$ (d, $J=2.3 \mathrm{~Hz}, 1 \mathrm{H}, \mathrm{ArH}), 5.31\left(\mathrm{~d}, J=4.6 \mathrm{~Hz}, 2 \mathrm{H}, \mathrm{CH}_{2}\right)$; ${ }^{13} \mathrm{C}$ NMR (DMSO- $\left.d_{6}, 75 \mathrm{MHz}\right) \delta: 182.57,164.10,163.58$, $162.54(\mathrm{~d}, J=244.5 \mathrm{~Hz}), 161.62,157.58,149.11,145.82$, $135.38(\mathrm{~d}, J=10.5 \mathrm{~Hz}), 132.67,132.02(\mathrm{~d}, J=9.0 \mathrm{~Hz})$, 130.98, 129.62 (2C), 126.92 (2C), 122.03 (d, $J=3.0 \mathrm{~Hz})$, $116.80(\mathrm{~d}, J=21.0 \mathrm{~Hz}), 113.51(\mathrm{~d}, J=24.8 \mathrm{~Hz}), 105.94$ (2C), 99.16, 94.20, 60.56; IR (KBr) v: 3066, 1656, 1614, $1512,1496,1448,1340,1155 \mathrm{~cm}^{-1}$; MS-MALDI 430 $\left[\mathrm{M}+\mathrm{H}^{+}\right]$; HRMS (ESI) calcd for $\mathrm{C}_{24} \mathrm{H}_{17} \mathrm{FN}_{3} \mathrm{O}_{4}\left[\mathrm{M}+\mathrm{H}^{+}\right]$ 430.1198, found 430.1192 .

7-((4-(2-Fluorophenyl)-4H-1,2,4-triazol-3-yl)methoxy)5-hydroxy-2-phenyl-4H-chromen-4-one (2d): Pale yellow solid, yield 14.5\%. m.p. $175 \sim 177{ }^{\circ} \mathrm{C} ;{ }^{1} \mathrm{H}$ NMR $\left(\mathrm{CDCl}_{3}\right.$, $300 \mathrm{MHz}) \delta: 12.71(\mathrm{~s}, 1 \mathrm{H}, \mathrm{OH}), 8.35(\mathrm{~s}, 1 \mathrm{H}$, Triazole-H), $7.90(\mathrm{dd}, J=7.8,1.7 \mathrm{~Hz}, 2 \mathrm{H}, \mathrm{ArH}), 7.54$ (dd, $J=7.2,3.4$ 
$\mathrm{Hz}, 4 \mathrm{H}, \mathrm{ArH}), 7.43 \sim 7.33(\mathrm{~m}, 3 \mathrm{H}, \mathrm{ArH}), 6.69(\mathrm{~s}, 1 \mathrm{H}$, ArH), $6.61(\mathrm{~d}, J=2.2 \mathrm{~Hz}, 1 \mathrm{H}), 6.20(\mathrm{~d}, J=2.2 \mathrm{~Hz}, 1 \mathrm{H}$, $\mathrm{ArH}), 5.33$ (d, $\left.J=7.1 \mathrm{~Hz}, 2 \mathrm{H}, \mathrm{CH}_{2}\right) ;{ }^{13} \mathrm{C} \mathrm{NMR}\left(\mathrm{CDCl}_{3}, 75\right.$ $\mathrm{MHz}) \delta: 182.48,164.28,162.95,162.54(\mathrm{~d}, J=133.5 \mathrm{~Hz})$, $162.09,157.69,149.73,145.04,141.64,132.12(\mathrm{~d}, J=8.3$ $\mathrm{Hz}), 132.03,131.04,129.13$ (2C), 127.94, 126.37 (2C), $125.32(\mathrm{~d}, J=3.8 \mathrm{~Hz}), 117.18(\mathrm{~d}, J=19.5 \mathrm{~Hz}), 106.45$, 105.95, 99.07, 92.91, 60.27; IR (KBr) v: 3066, 1654, 1612, 1514, 1496, 1448, 1338, $1157 \mathrm{~cm}^{-1}$; MS-MALDI $\mathrm{m} / \mathrm{z}: 430$ $\left[\mathrm{M}+\mathrm{H}^{+}\right]$; HRMS (ESI) calcd for $\mathrm{C}_{24} \mathrm{H}_{17} \mathrm{FN}_{3} \mathrm{O}_{4}\left[\mathrm{M}+\mathrm{H}^{+}\right]$ 430.1198, found 430.1199 .

7-((4-(4-Chlorophenyl)-4H-1,2,4-triazol-3-yl)methoxy)5-hydroxy-2-phenyl-4H-chromen-4-one (2e): Pale yellow solid, yield 25.5\%. m.p. 166 168 ${ }^{\circ} \mathrm{C}$; ${ }^{1} \mathrm{H}$ NMR (DMSO$\left.d_{6}, 300 \mathrm{MHz}\right) \delta: 12.81(\mathrm{~s}, 1 \mathrm{H}, \mathrm{OH}), 8.92(\mathrm{~s}, 1 \mathrm{H}$, Triazole-H), 8.08 (d, $J=8.0 \mathrm{~Hz}, 2 \mathrm{H}, \mathrm{ArH}), 7.64 \sim 7.29(\mathrm{~m}, 7 \mathrm{H}$, ArH), 7.04 (s, 1H, ArH), 6.84 (d, $J=2.1 \mathrm{~Hz}, 1 \mathrm{H}, \operatorname{ArH})$, $6.41(\mathrm{~d}, J=2.1 \mathrm{~Hz}, 1 \mathrm{H}, \mathrm{ArH}), 5.41\left(\mathrm{~s}, 2 \mathrm{H}, \mathrm{CH}_{2}\right) ;{ }^{13} \mathrm{C} \mathrm{NMR}$ $\left(\mathrm{CDCl}_{3}, 75 \mathrm{MHz}\right) \delta: 182.43,164.26,162.84,162.20$, $157.69,149.18,144.58,136.30,131.99,131.71,131.00$, 130.30 (2C), 129.09 (2C), 126.72 (2C), 126.34 (2C), 106.48, 105.94, 98.99, 93.10, 59.58; IR (KBr) v: 3107, $1656,1614,1504,1452,1350,1159 \mathrm{~cm}^{-1}$. MS-MALDI $m / z: 446\left[\mathrm{M}+\mathrm{H}^{+}\right]$; HRMS (ESI) calcd for $\mathrm{C}_{24} \mathrm{H}_{17} \mathrm{ClN}_{3} \mathrm{O}_{4}$ $\left[\mathrm{M}+\mathrm{H}^{+}\right]$446.0902, found 446.0907 .

7-((2-(3-Chlorophenyl)-2H-1,2,4-triazol-3-yl)methoxy)5-hydroxy-2-phenyl-4H-chromen-4-one (2f): Pale yellow solid, yield $22.1 \%$. m.p. $159 \sim 160{ }^{\circ} \mathrm{C} ;{ }^{1} \mathrm{H} \mathrm{NMR}\left(\mathrm{CDCl}_{3}\right.$, $300 \mathrm{MHz}) \delta: 12.75(\mathrm{~s}, 1 \mathrm{H}, \mathrm{OH}), 8.36(\mathrm{~s}, 1 \mathrm{H}$, Triazole-H), 7.90 (d, $J=8.1 \mathrm{~Hz}, 2 \mathrm{H}, \mathrm{ArH}), 7.56 \sim 7.46$ (m, 6H, ArH), 7.34 (s, 1H, ArH), 6.70 (d, J=2.6 Hz, 2H, ArH), 6.35 (d, $J=2.2 \mathrm{~Hz}, 1 \mathrm{H}, \mathrm{ArH}), 5.28\left(\mathrm{~s}, 2 \mathrm{H}, \mathrm{CH}_{2}\right) ;{ }^{13} \mathrm{C} \mathrm{NMR}\left(\mathrm{CDCl}_{3}\right.$, $75 \mathrm{MHz}) \delta: 182.45,164.31,162.34,144.44,135.87$, $134.39,131.96,131.10,130.33,129.11(2 \mathrm{C}), 126.37$ (2C), $125.90,123.63,113.03,106.58,106.04,99.10,93.24$, 77.41, 76.99, 76.57, 59.80; IR (KBr) v: 3111, 1660, 1618, $1502,1450,1350,1157 \mathrm{~cm}^{-1}$; MS-MALDI $m / z: 446[\mathrm{M}+$ $\mathrm{H}^{+}$]; HRMS (ESI) calcd for $\mathrm{C}_{24} \mathrm{H}_{17} \mathrm{ClN}_{3} \mathrm{O}_{4}\left[\mathrm{M}+\mathrm{H}^{+}\right]$ 446.0902, found 446.0905.

7-((4-(2-Chlorophenyl)-4H-1,2,4-triazol-3-yl)methoxy)5-hydroxy-2-phenyl-4H-chromen-4-one (2g): Pale yellow solid, yield 10.3\%. m.p. $157 \sim 158{ }^{\circ} \mathrm{C} ;{ }^{1} \mathrm{H}$ NMR $\left(\mathrm{CDCl}_{3}\right.$, $300 \mathrm{MHz}) \delta: 12.67(\mathrm{~s}, 1 \mathrm{H}, \mathrm{OH}), 8.31(\mathrm{~s}, 1 \mathrm{H}$, Triazole-H), $7.87(\mathrm{dd}, J=7.8,1.5 \mathrm{~Hz}, 2 \mathrm{H}, \operatorname{ArH}), 7.63 \sim 7.60(\mathrm{~m}, 1 \mathrm{H}$, ArH), $7.54 \sim 7.48(\mathrm{~m}, 4 \mathrm{H}, \mathrm{ArH}), 7.46 \sim 7.40(\mathrm{~m}, 2 \mathrm{H}, \mathrm{ArH})$, $6.66(\mathrm{~s}, 1 \mathrm{H}, \mathrm{ArH}), 6.57$ (d, J=2.2 Hz, 1H, ArH), 6.17 (d, $J=2.2 \mathrm{~Hz}, 1 \mathrm{H}, \mathrm{ArH}), 5.30\left(\mathrm{~d}, J=5.3 \mathrm{~Hz}, 2 \mathrm{H}, \mathrm{CH}_{2}\right) ;{ }^{13} \mathrm{C}$ NMR $\left(\mathrm{CDCl}_{3}, 75 \mathrm{MHz}\right) \delta$ : 182.44, 164.25, 162.99, 162.03, $157.66,149.67,144.91,132.01,131.84,131.50,131.06$, $131.00,130.78,129.11$ (2C), 128.83, 128.14, 126.35 (2C), $106.38,105.89$, 99.06, 92.86, 60.21; IR (KBr) v: 3103, 1654, 1618, 1504, 1454, 1346, $1157 \mathrm{~cm}^{-1}$; MS-MALDI $m / z: 446\left[\mathrm{M}+\mathrm{H}^{+}\right]$; HRMS (ESI) calcd for $\mathrm{C}_{24} \mathrm{H}_{17} \mathrm{ClN}_{3} \mathrm{O}_{4}$ $\left[\mathrm{M}+\mathrm{H}^{+}\right]$446.0902, found 446.0901.

7-((4-(3-Bromophenyl)-4H-1,2,4-triazol-3-yl)methoxy)5-hydroxy-2-phenyl-4H-chromen-4-one (2h): Pale yellow solid, yield $20.8 \%$. m.p. $179 \sim 181{ }^{\circ} \mathrm{C}$; ${ }^{1} \mathrm{H}$ NMR $\left(\mathrm{CDCl}_{3}\right.$,
$300 \mathrm{MHz}) \delta: 12.77$ (s, 1H, OH), 8.37 (s, 1H, Triazole-H), $7.92(\mathrm{~d}, J=8.2 \mathrm{~Hz}, 2 \mathrm{H}, \operatorname{ArH}), 7.70(\mathrm{~d}, J=8.3 \mathrm{~Hz}, 1 \mathrm{H}$, ArH), $7.63 \sim 7.55(\mathrm{~m}, 4 \mathrm{H}, \mathrm{ArH}), 7.48 \sim 7.38(\mathrm{~m}, 2 \mathrm{H}, \mathrm{ArH})$, $6.71(\mathrm{~s}, 2 \mathrm{H}, \mathrm{ArH}), 6.38(\mathrm{~d}, J=2.2 \mathrm{~Hz}, 1 \mathrm{H}, \mathrm{ArH}), 5.31(\mathrm{~s}$, $\left.2 \mathrm{H}, \mathrm{CH}_{2}\right) ;{ }^{13} \mathrm{C} \mathrm{NMR}\left(\mathrm{CDCl}_{3}, 75 \mathrm{MHz}\right) \delta: 182.46,164.32$, $162.91,162.35,157.77,149.18,144.44,134.47,133.27$, $131.96,131.23,131.15,129.11$ (2C), 128.80, 126.37 (2C), $124.10,123.49,106.59,106.04,99.11,93.25,59.80$; IR (KBr) v: 3107, 1660, 1618, 1500, 1452, 1350, $1157 \mathrm{~cm}^{-1}$; MS-MALDI $\mathrm{m} / \mathrm{z}: 490\left[\mathrm{M}+\mathrm{H}^{+}\right]$; HRMS (ESI) calcd for $\mathrm{C}_{24} \mathrm{H}_{17} \mathrm{BrN}_{3} \mathrm{O}_{4}\left[\mathrm{M}+\mathrm{H}^{+}\right]$490.0397, found 490.0393.

5-Hydroxy-2-phenyl-7-((4-p-tolyl-4H-1,2,4-triazol-3yl)methoxy)-4H-chromen-4-one (2i): Pale yellow solid, yield $25.4 \%$. m.p. $165 \sim 166{ }^{\circ} \mathrm{C}$; ${ }^{1} \mathrm{H}$ NMR $\left(\mathrm{CDCl}_{3}, 300\right.$ $\mathrm{MHz}) \delta: 12.81(\mathrm{~s}, 1 \mathrm{H}, \mathrm{OH}), 8.88(\mathrm{~s}, 1 \mathrm{H}$, Triazole-H), $8.09 \sim 8.06(\mathrm{~m}, 2 \mathrm{H}, \mathrm{ArH}), 7.64 \sim 7.57(\mathrm{~m}, 3 \mathrm{H}, \mathrm{ArH}), 7.41$ (dd, $J=28.2,8.2 \mathrm{~Hz}, 4 \mathrm{H}, \mathrm{ArH}), 7.05$ (s, 1H, ArH), 6.87 (d, $J=2.1 \mathrm{~Hz}, 1 \mathrm{H}, \mathrm{ArH}), 6.43(\mathrm{~d}, J=2.1 \mathrm{~Hz}, 1 \mathrm{H}, \mathrm{ArH}), 5.36$ $\left(\mathrm{s}, 2 \mathrm{H}, \mathrm{CH}_{2}\right), 2.35\left(\mathrm{~s}, 3 \mathrm{H}, \mathrm{CH}_{3}\right) ;{ }^{13} \mathrm{C} \mathrm{NMR}\left(\mathrm{CDCl}_{3}, 75\right.$ $\mathrm{MHz}) \delta$ : 182.32, 164.08, 163.02, 161.99, 157.56, 149.23, $144.73,140.27,131.88,130.90,130.48(2 \mathrm{C}), 128.99$ (2C), 126.21 (2C), 125.09 (2C), 106.25, 105.76, 98.99, 93.08, 90.29, 59.53, 21.09; IR (KBr) v: 3115, 1664, 1612, 1517, 1448, 1336, $1155 \mathrm{~cm}^{-1}$; MS-MALDI $\mathrm{m} / z: 426\left[\mathrm{M}+\mathrm{H}^{+}\right]$; HRMS (ESI) calcd for $\mathrm{C}_{25} \mathrm{H}_{20} \mathrm{~N}_{3} \mathrm{O}_{5}\left[\mathrm{M}+\mathrm{H}^{+}\right]$426.1448, found 426.1446 .

5-Hydroxy-2-phenyl-7-((4-m-tolyl-4H-1,2,4-triazol-3yl)methoxy)-4H-chromen-4-one (2j): Pale yellow solid, yield $15.4 \%$. m.p. $175 \sim 177{ }^{\circ} \mathrm{C} ;{ }^{1} \mathrm{H}$ NMR $\left(\mathrm{CDCl}_{3}, 300\right.$ $\mathrm{MHz}) \delta: 12.75(\mathrm{~s}, 1 \mathrm{H}, \mathrm{OH}), 8.37(\mathrm{~s}, 1 \mathrm{H}$, TriazoleH), $7.93 \sim 7.90(\mathrm{~m}, 2 \mathrm{H}, \mathrm{ArH}), 7.56(\mathrm{~d}, J=7.3 \mathrm{~Hz}, 3 \mathrm{H}, \mathrm{ArH})$, $7.46 \sim 7.34(\mathrm{~m}, 2 \mathrm{H}, \mathrm{ArH}), 7.23(\mathrm{~s}, 2 \mathrm{H}, \mathrm{ArH}), 6.72 \sim 6.71$ (m, 2H, ArH), 6.35 (d, J=2.2 Hz, 1H, ArH), $5.28(\mathrm{~s}, 2 \mathrm{H}$, $\left.\mathrm{CH}_{2}\right), 2.44\left(\mathrm{~s}, 3 \mathrm{H}, \mathrm{CH}_{3}\right) ;{ }^{13} \mathrm{C} \mathrm{NMR}\left(\mathrm{CDCl}_{3}, 75 \mathrm{MHz}\right) \delta$ : $182.51,164.29,163.14,162.18,157.75,153.43,144.73$, $142.39,140.45,132.01,131.09,130.80,129.82,129.13$ (2C), 126.38 (2C), 126.01, 122.38, 106.44, 105.97, 99.15, 93.21, 59.69, 21.32; IR (KBr) v: 3072, 1658, 1618, 1502, 1450, 1350, $1159 \mathrm{~cm}^{-1}$; MS-MALDI $m / z: 426\left[\mathrm{M}+\mathrm{H}^{+}\right]$; HRMS (ESI) calcd for $\mathrm{C}_{25} \mathrm{H}_{20} \mathrm{~N}_{3} \mathrm{O}_{5}\left[\mathrm{M}+\mathrm{H}^{+}\right]$426.1448, found 426.1446 .

5-Hydroxy-7-((4-(4-methoxyphenyl)-4H-1,2,4-triazol-3yl)methoxy)-2-phenyl-4H-chromen-4-one (2k): Pale yellow solid, yield $24.9 \%$. m.p. $163 \sim 165{ }^{\circ} \mathrm{C} ;{ }^{1} \mathrm{H}$ NMR $\left(\mathrm{DMSO}-d_{6}, 300 \mathrm{MHz}\right) \delta: 12.82(\mathrm{~s}, 1 \mathrm{H}, \mathrm{OH}), 8.85(\mathrm{~s}, 1 \mathrm{H}$, Triazole-H), 8.10 8.07 (m, 2H, ArH), $7.62 \sim 7.59(\mathrm{~m}, 3 \mathrm{H}$, ArH), $7.50(\mathrm{~d}, J=8.9 \mathrm{~Hz}, 2 \mathrm{H}, \mathrm{ArH}), 7.11 \sim 7.06(\mathrm{~m}, 3 \mathrm{H}$, ArH), $6.87(\mathrm{~d}, J=2.1 \mathrm{~Hz}, 1 \mathrm{H}, \mathrm{ArH}), 6.44(\mathrm{~d}, J=2.1 \mathrm{~Hz}$, $1 \mathrm{H}, \mathrm{ArH}), 5.34\left(\mathrm{~s}, 2 \mathrm{H}, \mathrm{CH}_{2}\right), 3.79\left(\mathrm{~s}, 3 \mathrm{H}, \mathrm{CH}_{3}\right) ;{ }^{13} \mathrm{C} \mathrm{NMR}$ $\left(\mathrm{CDCl}_{3}, 75 \mathrm{MHz}\right) \delta: 182.31,164.08,163.03,161.99$, $160.50,157.57,149.43,144.92,131.86,130.90,128.98$ (2C), 126.73 (2C), 126.21 (2C), 125.70, 114.95 (2C), 106.25, 105.77, 98.99, 93.06, 59.52, 55.57; IR (KBr) v: $3078,1658,1616,1517,1452,1348,1157 \mathrm{~cm}^{-1}$; MS-MALDI $442\left[\mathrm{M}+\mathrm{H}^{+}\right]$; HRMS (ESI) calcd for $\mathrm{C}_{25} \mathrm{H}_{20} \mathrm{~N}_{3} \mathrm{O}_{5}\left[\mathrm{M}+\mathrm{H}^{+}\right]$442.1398, found 442.1396. 


\subsection{Biological testing}

The antiproliferative activities of the target compounds against MGC-803, BEL-7402, HepG2, HeLa, A549, and SGC-7901 cell lines were evaluated by MTT assay in vitro, with 5-FU as the positive control. All cells were cultured in RPMI 1640 medium supplemented with $10 \%$ fetal bovine serum (FBS). Cells were detached by trypsin, seeded at $9 \times 10^{3}$ cells in a 96-well plate and incubated in $5 \% \mathrm{CO}_{2}$ at $37{ }^{\circ} \mathrm{C}$ overnight. Then the cells are treated with the test compounds at different concentrations $(1,10$, 50, and $100 \mu \mathrm{mol} / \mathrm{L}$ ) and incubated for $48 \mathrm{~h}$. Fresh MTT solutions were added to each well and incubated at $37{ }^{\circ} \mathrm{C}$ for $4 \mathrm{~h}$. The MTT-formazan formed by metabolically viable cells was dissolved by dimethyl sulfoxide (DMSO, 150 $\mu \mathrm{L}$ ) in each well and monitored by a microplate reader at a wavelength of $492 \mathrm{~nm}$. $\mathrm{IC}_{50}$ was defined as the drug concentrations that inhibited the cell number to $50 \%$ after 48 h.

Supporting Information NMR spectra of target compounds. The Supporting Information is available free of charge via the Internet at http://sioc-journal.cn/.

\section{References}

[1] Chen, Y. H.; Yang, Z. S.; Wen, C. C.; Chang, Y. S.; Wang, B. C.; Hsiao, C. A.; Shih, T. L. Food Chem. 2012, 134, 717.

[2] Critchfield, J. W.; Butera, S. T.; Folks, T. M. AIDS Res. Hum. Retroviruses 1996, 12, 39.
[3] Du, Q.; Gu, X.; Cai, J.; Huang, M.; Su, M. Mol. Med. Rep. 2012, 6, 100.

[4] Gresa-Arribas, N.; Serratosa, J.; Saura, J.; Solã, C. J. Neurochem. 2010, 115, 526.

[5] Brown, E.; Hurd, N. S.; McCall, S.; Ceremuga, T. E. AANA J. 2007, 75,333 .

[6] Khoo, B. Y.; Chua, S. L.; Balaram, P. Int. J. Mol. Sci. 2010, 11, 2188.

[7] Prabhakar, M. M.; Manoharan, S.; Baskaran, N.; Srinivasan, R.; Karthikeyan, S.; Wani, S. A. Int. J. Phram. Sci. 2012, 3, 89.

[8] Srinivasan, R.; Manoharan, S. J. Cell Tissue Res. 2011, 11, 2909.

[9] Yu, X. M.; Phan, T.; Patel, P. N.; Jaskula-Sztul, R.; Chen, H. Cancer 2013, 119, 774

[10] Tsuji, P. A.; Winn, R. N.; Walle, T. Chem. Biol. Interact. 2006, 164, 85.

[11] Walle, U. K.; Galijatovic, A.; Walle, T. Biochem. Pharmacol. 1999, 58,431 .

[12] Dos Anjos, J. V.; Sinou, D.; De Melo, S. J.; Srivastava, R. M. Carbohydr. Res. 2007, 342, 2440.

[13] Luan, T.; Cao, L. H.; Deng, H.; Shen, Q. K.; Tian, Y. S.; Quan, Z. S. Molecules 2019, 24, 121.

[14] Liu, C. F.; Shen, Q. K.; Li, J. J.; Tian, Y. S.; Quan, Z. S. J. Enzym. Inhib. Med. Chem. 2017, 32, 1111.

[15] Hu, K.; Wang, W.; Cheng, H.; Pan, S. S.; Ren, J. Med. Chem. Res. 2011, 20, 838 .

[16] Liu, J. W.; Taylor, S. F.; Dupart, P. S.; Arnold, C. L.; Sridhar, J.; Jiang, Q.; Wang, Y.; Skripnikova, E. V.; Zhao, M.; Foroozesh, M. J. Med. Chem. 2013, 56, 4082.

[17] Maingot, L.; Elbakali, J.; Dumont, J.; Bosc, D.; Cousaert, N.; Urban, A.; Deglane G.; Villoutreix, B.; Nagase, H.; Sperandio, O.; Leroux, F.; Deprez, B.; Deprez-Poulain, R. Eur. J. Med. Chem. 2013, 69, 244.

[18] Zou, X. Q.; Peng, S. M.; Hu, C. P.; Tan, L. F.; Yuan, Q.; Deng, H. W.; Li, Y. J. Bioorg. Med. Chem. 2010, 18, 3020.

(Cheng, F.) 